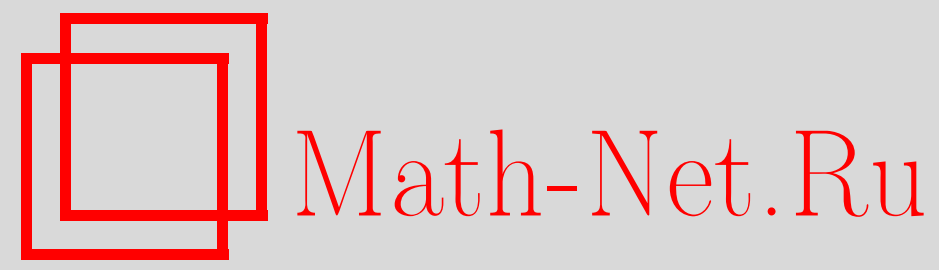

Д. А. Коршунов, Вероятности больших уклонений максимумов сумм независимых слагаемых с отрицательным средним и субэкспоненциальным распределением, Теория вероятн. и ее примен., 2001, том 46, выпуск 2, 387-397

DOI: https://doi.org/10.4213/tvp3929

Использование Общероссийского математического портала MathNet.Ru подразумевает, что вы прочитали и согласны с пользовательским соглашением

http://www . mathnet.ru/rus/agreement

Параметры загрузки:

IP: 18.234 .197 .8

26 апреля 2023 г., 18:06:59

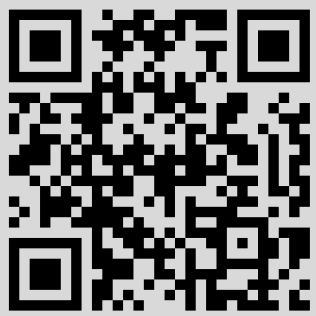


(C) $2001 \mathrm{r}$.

КОРшУНОВ Д. А.*

\title{
ВЕРОЯТНОСТИ БОЛЬШИХ УКЛОНЕНИЙ МАКСИМУМОВ СУММ НЕЗАВИСИМЫХ СЛАГАЕМЫХ С ОТРИЦАТЕЛЬНЫМ СРЕДНИМ И СУБЭКСПОНЕНЦИАЛЬНЫМ РАСПРЕДЕЛЕНИЕМ ${ }^{1)}$
}

\begin{abstract}
Рассматриваются суммы $S_{n}=\xi_{1}+\cdots+\xi_{n}$ независимых одинаково распределенных случайных величин с отрицательным средним значением. В случае сильно субэкспоненциального распределения слагаемых найдена асимптотика вероятности того, что максимум сумм $\max \left(S_{1}, \ldots, S_{n}\right)$ превзойдет большой уровень $x$. Полученные утверждения об асимптотике этой вероятности имеют равномерный по всем значениям $n$ характер.
\end{abstract}

Ключевые слова и фразы: максимумы сумм случайных величин, однородная цепь Маркова, вероятности больших уклонений, субэкспоненциальное распределение, второй хвост распределения.

1. Введение. Пусть $\xi, \xi_{1}, \xi_{2}, \ldots$ - независимые случайные величины с общим распределением $F$ на вешественной прямой $\mathbf{R} ; F((-\infty, 0])<1$. Обозначим $F(x)=$ $F((-\infty, x)), \bar{F}(x)=1-F(x)$. Вообще, для любой меры $G$ через $\bar{G}(x)=G([x, \infty))$ обозначаем хвост этой меры. Положим $S_{0}=0, S_{n}=\xi_{1}+\cdots+\xi_{n}$ и

$$
M_{n}=\max \left\{S_{k}, 0 \leqslant k \leqslant n\right\} .
$$

Предполагаем, что существует $\mathbf{E} \boldsymbol{\xi}$, причем $\mathbf{E} \boldsymbol{\xi}<0$; положим $\boldsymbol{a}=|\mathbf{E} \boldsymbol{\xi}|$. По усиленному закону больших чисел $S_{n} \rightarrow-\infty$ при $\boldsymbol{n} \rightarrow \infty$ почти наверное, и, следовательно, семейство распределений максимумов $M_{n}, n \geqslant 1$, слабо компактно.

Основной задачей настоящей работы является изучение асимптотического поведения вероятности $\mathbf{P}\left\{M_{n} \geqslant x\right\}$ при $x \rightarrow \infty$ в случае, когда отдельное слагаемое имеет распределение $F$ типа субэкспоненциального; нас интересуют как фиксированные значения временного параметра $n$, так и случай неограниченно возрастающего $n$. Точнее, будут получены утверждения об асимптотическом поведении вероятности $\mathbf{P}\left\{M_{n} \geqslant x\right\}$, равномерные по $n$.

Напомним определения некоторых классов функций и распределений, используемых в дальнейшем изложении.

О п р е д е л е н и е 1. Функция $f$ называется лохальяо степеняой, если для всякого фиксированного $t$ отношение $f(x+t) / f(x)$ стремится к 1 при $x \rightarrow \infty$. Говорим, что распределение $G$ локально степенное, если функция $\bar{G}(x)$ локально степенная.

О п р е д е л е н и е 2. Говорят, что распределение $G$ в $\mathbf{R}^{+}$с неограниченным носителем принадлежит классу $\mathscr{S}$ (является субзкспоненциальныљ распределением), если хвост свертки $\overline{G * G}(x)$ эквивалентен $2 \bar{G}(x)$ при $x \rightarrow \infty$.

В [4] показано, что субэкспоненциальное распределение $G$ обязательно локально степенное. Достаточные условия принадлежности распределения классу $\mathscr{S}$ можно найти, например, в [4], [10]. Класс $\mathscr{S}$ включает в себя, в частности, следующие распределения: (а) распределение Парето с хвостом $\bar{G}(x)=(\varkappa / x)^{\alpha}, x \geqslant \varkappa$, где $\varkappa>0$, $\alpha>0$; (б) логнормальное распределение с плотностью $e^{-(\ln x-\ln \alpha)^{2} / 2 \sigma^{2}} / x \sigma \sqrt{2 \pi}$, $x>0$, где $\sigma>0, \alpha>0$; (в) распределение Вейбулла с хвостом $\bar{G}(x)=e^{-x^{\alpha}}, x \geqslant 0$, где $\alpha \in(0,1)$.

* Институт математики им. С. Л. Соболева СО РАН, просп. Академика Коптюга, 4, 630090 Новосибирск, Россия; e-mail: korshunov@math.nsc.ru

1) Работа выполнена при частичной финансовой поддержке Российского фонда фундаментальных исследований (проекты № 99-01-01504 и № 99-01-01561) и CRDF Cooperative Grants Program (Award \# RM1-226) и INTAS (Project № 93-10820). 
Пусть $G$ - произвольное распределение в $\mathbf{R}$ с неограниченным справа носителем и конечным средним значением. Для любого $t>0$ введем в рассмотрение распределение $G_{t}$ в $\mathbf{R}^{+}$такое, что

$$
\overline{G_{t}}(x)=\min \left(1, \int_{x}^{x+t} \bar{G}(u) d u\right), \quad x>0
$$

(здесь и всюду в дальнейшем под интегралом в пределах от $x_{1}$ до $x_{2}$ понимается интеграл по множеству $\left.\left[x_{1}, x_{2}\right)\right)$. Семейство распределений $\left\{G_{t}, t>0\right\}$ является стохастически возрастающим семейством.

O п р е д е л е н и е 3 . Будем говорить, что субэкспоненциальное распределение $G$ является сиљьяо субэкспоненциалькым (и писать $G \in \mathscr{S}_{*}$ ), если $\overline{G_{t} * G_{t}}(x) / \overline{G_{t}}(x) \longrightarrow 2$ при $x \rightarrow \infty$ равномерно по $t \in[1, \infty]$.

По определению $\mathscr{S}_{*} \subseteq \mathscr{S}$. Возможно класс $\mathscr{S}_{*}$ совпадает с классом субэкспоненциальных распределений с конечным средним значением, однако мы не располагаем доказательством этого факта. Как отмечается в [7], неясно даже влечет ли принадлежность $G \in \mathscr{S}$ субэкспоненциальность распределения $G_{\infty}$. Достаточные условия принадлежности распределения классу $\mathscr{S}_{*}$ приведены в п. 3. Там же проверено, что распределения Парето (при $\alpha>1$ ), логнормальное и Вейбулла удовлетворяют этим условиям и, следовательно, являются сильно субэкспоненциальными.

Определим случайную последовательность $X=\left\{X_{n}\right\}$ равенством

$$
X_{n+1}=\left(X_{n}+\xi_{n+1}\right)^{+} \text {. }
$$

Она образует однородную по времени цепь Маркова, являющуюся случайным блужданием с задержкой в нуле. Хорошо известно (см., например, [3, гл. VI, §9]), что распределение цепи $X$ в момент времени $n$ при нулевом начальном условии $X_{0}=0$ совпадает с распределением $M_{n}$, т.e.

$$
\mathbf{P}\left\{M_{n} \geqslant x\right\}=\mathbf{P}\left\{X_{n} \geqslant x \mid X_{0}=0\right\} .
$$

Поэтому изучение асимптотики вероятности $\mathbf{P}\left\{M_{n} \geqslant x\right\}$ равносильно изучению асимптотики вероятности $\mathbf{P}\left\{X_{n} \geqslant x \mid X_{0}=0\right\}$. Отметим, что конечномерные распределения последовательностей $\left\{M_{n}\right\}$ и $\left\{X_{n}\right\}$ не совпадают.

В работе [11] доказано, что если распределение $F_{\infty}$ на полупрямой $\mathbf{R}^{+}$является субэкспоненциальным, то хвост распределения супремума сумм эквивалентен второму хвосту распределения отдельного слагаемого, т.е. при $x \rightarrow \infty$

$$
\mathbf{P}\left\{\sup _{n \geqslant 1} S_{n} \geqslant x\right\} \sim \frac{1}{a} \int_{x}^{\infty} \bar{F}(u) d u .
$$

В [8] показано, что субэкспоненциальность распределения $F_{\infty}$ является необходимым условием для справедливости асимптотики (4).

В [9] рассмотрен случай фиксированного значения $\boldsymbol{n}$ и доказано, что если распределение случайной величины $\boldsymbol{\xi} \mathbf{I}\{\xi \geqslant 0\}$ субэкспоненциальное, то $\mathbf{P}\left\{M_{n} \geqslant x\right\} \sim n \bar{F}(x)$ при $x \rightarrow \infty$.

В настоящей работе показано, что имеет место следующая

Теорема. Пусть распределение случайной величияы $\xi \mathbf{I}\{\xi \geqslant 0\}$ сильно субэкспонекциалькое. Тогда

$$
\mathbf{P}\left\{M_{n} \geqslant x\right\}=\frac{1+\varepsilon_{n}(x)}{a} \int_{x}^{x+n a} \bar{F}(u) d u,
$$

где $\varepsilon_{n}(x) \rightarrow 0$ при $x \rightarrow \infty$ равномерко по $n \geqslant 1$.

Утверждение теоремы вытекает из лемм 1 и 9 , доказанных в пा. 2 и 6 соответственно.

Как нам стало известно, после написания настоящей работы аналогичная эквивалентность установлена другими методами в [5, теорема 6] в случае, когда $\mathbf{E} \xi^{2}<\infty$ и функция $\bar{F}(u)$ правильно меняется на бесконечности.

Относительно более ранних результатов следует отметить статьи [2] и [1]. В работе [2] рассмотрен случай, когда функция $\bar{F}(x)$ правильно меняется на бесконечности с показателем $\alpha \in(-\infty,-2)$. Полагая присутствующую в теореме 2 этой 
работы функцию $g(t)$ равной $1+a t / \gamma$, а $x$ равным $\gamma n$, можно вывести оттуда для любого фиксированного $\gamma>0$ аскмптотику

$$
\mathbf{P}\left\{S_{k} \geqslant \gamma \boldsymbol{n} \text { для некоторого } k \leqslant n\right\} \sim n \mathbf{P}\{\xi \geqslant \gamma n\} c_{\alpha},
$$

где $c_{\alpha}=\int_{0}^{1}[g(t)]^{-\alpha} d t$. Эта асимптотика совпадает с предлагаемой нами, если положить $x=\gamma n$.

Такая же, как и в [2], форма ответа в случае $\alpha \in(-2,-1)$ приведена в [1].

2. Оценка снизу для вероятностей больших уклонений максимума сумм. В следующей лемме при минимальных ограничениях на распределение $F$ вероятность $\mathbf{P}\left\{M_{n} \geqslant x\right\}$ оценивается снизу при больших значениях $\boldsymbol{x}$.

Лемма 1. Пусть распределение $F$ лохалько степекное. Тогда для мюбого $\varepsilon>0$ найдется $x_{1}$ такое, что при $x \geqslant x_{1}$ u $n \geqslant 1$ справедливо керавекство

$$
\mathbf{P}\left\{M_{n} \geqslant x\right\} \geqslant \frac{1-\varepsilon}{a} \int_{x}^{x+n a} \bar{F}(u) d u .
$$

Д о к а з а те л ь т в о. Ввиду равенства (3) достаточно доказать соответствующее утверждение для цепи (2) с нулевым начальным состоянием $X_{0}=0$. Как отмечалось выше, семейство максимумов $\left\{M_{n}, n \geqslant 1\right\}$ ограничено по вероятности. Поэтому семейство $\left\{X_{n}, n \geqslant 1\right\}$ также ограничено по вероятности, т.е. имеет место сходимость

$$
\inf _{n \geqslant 1} \mathbf{P}\left\{X_{n}<x\right\} \longrightarrow 1 \quad \text { при } x \rightarrow \infty \text {. }
$$

Рассмотрим событие $A_{i n}, i \in[1, n]$, состоящее в том, что $X_{i-1}<x$ и $X_{j} \geqslant x$ для любого $j \in[i, n]$. Поскольку события $A_{i n}, i \in[1, n]$, не пересекаются, а их объединение равно событию $\left\{X_{n} \geqslant x\right\}$, то по формуле полной вероятности

$$
\mathbf{P}\left\{X_{n} \geqslant x\right\}=\mathbf{P}\left\{A_{1 n}\right\}+\cdots+\mathbf{P}\left\{A_{n n}\right\} .
$$

Пусть $\delta>0$; положим $b=a+\delta$. Для $v \geqslant 0$ введем вероятность $p_{i}(x+v)$ равенством

$$
p_{i}(x+v)=\mathbf{P}\left\{X_{j} \geqslant x \text { для любого } j \leqslant i \mid X_{0}=x+v\right\} .
$$

Ввиду определения (2) при начальном состоянии $X_{0}=x+v$ и любом $i$ справедливо неравенство $X_{i} \geqslant x+v+\xi_{1}+\cdots+\xi_{i}$. Поэтому $p_{i}(x+v) \geqslant \mathbf{P}\left\{v+\xi_{1}+\cdots+\xi_{j} \geqslant 0\right.$ для любого $j \leqslant i\}$. Полагая здесь $v=U+i b$, по усиленному закону больших чисел получаем равномерную по $x$ и $i$ сходимость

$$
p_{i}(x+U+i b) \rightarrow 1 \quad \text { при } U \rightarrow \infty .
$$

Так как совместное наступление событий $\left\{X_{i-1}<x\right\},\left\{X_{i} \geqslant x+U+(n-i) b\right\}$ и $\left\{X_{j} \geqslant x\right.$ при $\left.j \in[i+1, n]\right\}$ влечет событие $A_{i n}$, то справедливо неравенство

$$
\mathbf{P}\left\{A_{i n}\right\} \geqslant \int_{0}^{x} \mathbf{P}\left\{X_{i-1} \in d y\right\} \int_{U+(n-i) b}^{\infty} \mathbf{P}\left\{X_{i} \in x+d u \mid X_{i-1}=y\right\} p_{n-i}(x+u) .
$$

Поскольку функция $p_{i}(x+u)$ не убывает по $u$,

$$
\begin{aligned}
\mathbf{P}\left\{A_{i n}\right\} \geqslant & p_{n-i}(x+U+(n-i) b) \\
& \times \int_{0}^{x} \mathbf{P}\left\{X_{i-1} \in d y\right\} \mathbf{P}\left\{X_{i} \geqslant x+U+(n-i) b \mid X_{i-1}=y\right\} .
\end{aligned}
$$

Учитывая, что при любом $y \in[0, x)$

$$
\mathbf{P}\left\{X_{i} \geqslant x+U+(n-i) b \mid X_{i-1}=y\right\} \geqslant \mathbf{P}\{\xi \geqslant x+U+(n-i) b\},
$$

из последнего неравенства получаем

$$
\mathbf{P}\left\{A_{i n}\right\} \geqslant p_{n-i}(x+U+(n-i) b) \mathbf{P}\left\{X_{i-1}<x\right\} \mathbf{P}\{\xi \geqslant x+U+(n-i) b\} .
$$

В силу этой оценки и сходимости (7) найдется достаточно большое $U$ такое, что при всех $x, i$ и $n$ справедливо неравенство $\mathbf{P}\left\{A_{i n}\right\} \geqslant(1-\delta / 2) \mathbf{P}\left\{X_{i-1}<x\right\} \mathbf{P}\{\boldsymbol{\xi} \geqslant$ $x+U+(n-i) b\}$. Отсюда ввиду сходимости (5) при достаточно больших $x$

$$
\mathbf{P}\left\{A_{\text {in }}\right\} \geqslant(1-\delta) \bar{F}(x+U+(n-i) b)
$$


равномерно по $i$ и $n$. Используя последнее неравенство, выводим из равенства (6) оценку, верную для достаточно больших $x$ :

$$
\mathbf{P}\left\{X_{n} \geqslant x\right\} \geqslant(1-\delta) \sum_{i=1}^{n} \bar{F}(x+U+(n-i) b) .
$$

Так как функция $\bar{F}(v)$ локально степенная, то равномерно по $n$

$$
\sum_{i=1}^{n} \bar{F}(x+U+(n-i) b) \sim \frac{1}{b} \int_{x}^{x+n b} \bar{F}(v) d v \quad \text { при } x \rightarrow \infty \text {. }
$$

Поскольку $b=a+\delta$ и $\delta>0$ выбрано произвольно, отсюда вытекает утверждение леммы.

3. Условия принадлежности распределения классу $\mathscr{S}_{*}$. Пусть $G-$ локально степенное распределение в $\mathbf{R}^{+}$с конечным средним значением. Для любого $U \in(0, x)$ имеем равенства (распределение $G_{t}$ определено в $\left.(1)\right)$ :

$$
\begin{aligned}
\frac{\overline{G_{t} * G_{t}}(x)}{\overline{G_{t}}(x)} & =\int_{0}^{x} \frac{\overline{G_{t}}(x-u)}{\overline{G_{t}}(x)} d G_{t}(u)+1 \\
& =\left(\int_{0}^{U}+\int_{U}^{x}\right) \frac{\overline{G_{t}}(x-u)}{\overline{G_{t}}(x)} d G_{t}(u)+1 .
\end{aligned}
$$

Так как функция $\overline{G_{t}}(y)$ локально степенная, то при любом фиксированном $U$

$$
\int_{0}^{U} \frac{\overline{G_{t}}(x-u)}{\overline{G_{t}}(x)} d G_{t}(u) \longrightarrow G_{t}(U)
$$

при $x \rightarrow \infty$ равномерно по $t \geqslant 1$. Следовательно, имеет место

Лемма 2. Пусть распределение $G$ - лохалько степенное. Тогда следующие условия эквивалентны:

(i) $G \in \mathscr{S}_{*}$;

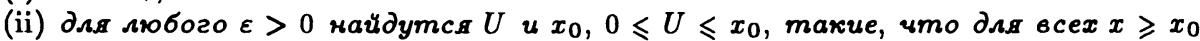
$u t \geqslant 1$ выполняется

$$
\int_{U}^{x} \overline{G_{t}}(x-u) d G_{t}(u) \leqslant \varepsilon \overline{G_{t}}(x)
$$

(iii) существует функиия $U(x) \rightarrow \infty, 0 \leqslant U(x) \leqslant x$, такая, что $\bar{G}(x-U(x)) \sim$ $\bar{G}(x)$ и равкомерно по $t \geqslant 1$

$$
\int_{U(x)}^{x} \overline{G_{t}}(x-u) d G_{t}(u)=o\left(\overline{G_{t}}(x)\right) \quad \text { npu } x \rightarrow \infty
$$

(iv) для яюбой функчии $U(x) \rightarrow \infty, 0 \leqslant U(x) \leqslant x$, maкой, что $\bar{G}(x-U(x)) \sim \bar{G}(x)$, выполняется равномерное по $t \geqslant 1$ соотношекие

$$
\int_{U(x)}^{x} \overline{G_{t}}(x-u) d G_{t}(u)=o\left(\overline{G_{t}}(x)\right) \quad \text { nрu } x \rightarrow \infty .
$$

В следующей лемме формулируется свойство замкнутости класса $\mathscr{S}_{*}$ относительно отношения слабой эквивалентности хвостов распределений (терминология из [7]).

Лемма 3. Пусть $G$ и $H$ - два локальяо степенных распределения в $\mathbf{R}^{+}$. Если $G \in \mathscr{S}_{*} u c_{1} \bar{G}(x) \leqslant \bar{H}(x) \leqslant c_{2} \bar{G}(x)$ длs некоторых $c_{1} u c_{2}, 0<c_{1}<c_{2}<\infty$, mo $H \in \mathscr{S}_{*}$.

Д ок а з а те л ь с в о. Так как распределение $H$ локально степенное, а $G$ сильно субэкспоненциальное, по лемме 2 найдется последовательность $U(x) \rightarrow \infty$ такая, что $\bar{G}(x-U(x)) \sim \bar{G}(x), \bar{H}(x-U(x)) \sim \bar{H}(x)$ и

$$
\int_{U(x)}^{x} \overline{G_{t}}(x-u) d G_{t}(u)=o\left(\overline{G_{t}}(x)\right)
$$


при $x \rightarrow \infty$ равномерно по $t \geqslant 1$. Поскольку $\bar{H}(x) \leqslant c_{2} \bar{G}(x)$, то после интегрирования по частям получаем

$$
\begin{aligned}
& \int_{U(x)}^{x} \overline{H_{t}}(x-u) d H_{t}(u) \leqslant c_{2} \int_{U(x)}^{x} \overline{G_{t}}(x-u) d H_{t}(u) \\
& \quad=-\left.c_{2} \overline{G_{t}}(x-u) \overline{H_{t}}(u)\right|_{U(x)} ^{x}+c_{2} \int_{U(x)}^{x} \overline{H_{t}}(u) d_{u} \overline{G_{t}}(x-u) \\
& \quad \leqslant c_{2} \overline{G_{t}}(x-U(x)) \overline{H_{t}}(U(x))+c_{2}^{2} \int_{U(x)}^{x} \overline{G_{t}}(u) d_{u} \overline{G_{t}}(x-u) .
\end{aligned}
$$

Отсюда ввиду $(10)$ и условия $\bar{H}(x) \geqslant c_{1} \bar{G}(x)$ вытекает соотношение

$$
\int_{U(x)}^{x} \overline{H_{t}}(x-u) d H_{t}(u)=o\left(\overline{G_{t}}(x)\right)=o\left(\overline{H_{t}}(x)\right),
$$

которое в силу леммы 2 завершает доказательство.

Лемма 4. Пусть распределение $G$ с конечным средним зяачением является субэкспоненциальным и существует $c>0$ тахое, ито $\bar{G}(2 x) \geqslant c \bar{G}(x)$ для любого $x$. Тогда распределение $G$ вөляется сильно субэкспокекциальным.

Д ок а з а т е л с с т в. Имеем

$$
\begin{aligned}
\overline{G_{t}}(2 x) & =\min \left(1, \int_{2 x}^{2 x+t} \bar{G}(u) d u\right) \\
& =\min \left(1,2 \int_{x}^{x+t / 2} \bar{G}(2 u) d u\right) \geqslant \min \left(1, \int_{x}^{x+t} \bar{G}(2 u) d u\right) .
\end{aligned}
$$

Так как $\bar{G}(2 u) \geqslant c \bar{G}(u)$, то отсюда вытекает неравенство

$$
\overline{G_{t}}(2 x) \geqslant \min \left(c, c \int_{x}^{x+t} \bar{G}(u) d u\right)=c \overline{G_{t}}(x) .
$$

Кроме того, функция $\bar{G}(y)$ локально степенная. Поэтому для любого фиксированного $u$ равномерно по $t \geqslant 1$

$$
\overline{G_{t}}(x-u)\left[\overline{G_{t}}(x)\right]^{-1} \longrightarrow 1 \quad \text { при } x \rightarrow \infty .
$$

Для хвоста свертки $G_{t} * G_{t}$ с помощью интегрирования по частям и с учетом непрерывности распределения $G_{t}$ в точках $u>0$ получаем выражения:

$$
\begin{aligned}
\overline{G_{t} * G_{t}}(x) & =-\left(\int_{0}^{x / 2}+\int_{x / 2}^{\infty}\right) \overline{G_{t}}(x-u) d \overline{G_{t}}(u) \\
& =\int_{0}^{x / 2} \overline{G_{t}}(x-u) d G_{t}(u)+\int_{x / 2}^{\infty} \overline{G_{t}}(u) d_{u} \overline{G_{t}}(x-u)+\left(\overline{G_{t}}\left(\frac{x}{2}\right)\right)^{2} \\
& =2 \int_{0}^{x / 2} \overline{G_{t}}(x-u) d G_{t}(u)+\left(\overline{G_{t}}\left(\frac{x}{2}\right)\right)^{2}
\end{aligned}
$$

Рассмотрим интеграл

$$
\int_{0}^{x / 2} \frac{\overline{G_{t}}(x-u)}{\overline{G_{t}}(x)} d G_{t}(u)=\left(\int_{0}^{U}+\int_{U}^{x / 2}\right) \frac{\overline{G_{t}}(x-u)}{\overline{G_{t}}(x)} d G_{t}(u) .
$$

Ввиду (12) первое слагаемое для любого фиксированного $U$ имеет своим пределом $G_{t}(U)$. В силу (11) второе слагаемое не превосходит $\overline{G_{\infty}}(U) / c$ и выбором достаточно большого $U$ может быть сделано сколь угодно малым. Из сказанного вытекает равномерная по $t \geqslant 1$ сходимость интегралов

$$
\int_{0}^{x / 2} \frac{\overline{G_{t}}(x-u)}{\overline{G_{t}}(x)} d G_{t}(u) \longrightarrow 1 \quad \text { при } x \rightarrow \infty .
$$

Из (11) вытекает также, что $\left(\overline{G_{t}}(x / 2)\right)^{2} \leqslant \overline{G_{t}}(x) \overline{G_{t}}(x / 2) / c=o\left(\overline{G_{t}}(x)\right)$ при $x \rightarrow \infty$. Подставляя два последние соотношения в (13), приходим к требуемой равномерной по $t \geqslant 1$ асимптотике $\overline{G_{t} * G_{t}}(x) \sim 2 \overline{G_{t}}(x)$ при $x \rightarrow \infty$. 
Лемма 5. Пусть распределение $G$ с конечным средним значением ввляется субэкспоненииальньм. Пусть существует $x_{0}$ такое, ито функчия $g(x) \equiv-\ln \bar{G}(x)$ вогнута при $x \geqslant x_{0}$ u, кроме того,

$$
\int_{0}^{x} \bar{G}(x-u) \bar{G}(u) d u \sim \bar{G}(x) \int_{0}^{\infty} \bar{G}(u) d u \quad \text { при } x \rightarrow \infty .
$$

Тогда распределение $G$ двляется сильно субэкспоненчиальным.

Д о к а $з$ а т е л ь с т в о. Обозначим $J_{t}(x)=\bar{G}_{t}(x) / \bar{G}(x)$. В частности, если случайная величина $\zeta$ имеет распределение $G$, то $J_{\infty}(x)=\mathbf{E}\{\zeta \mid \zeta \geqslant x\}$ для таких значений $x$, что $\overline{G_{\infty}}(x) \leqslant 1$.

Так как функция $g(x)$ вогнута, разность $g(x+u)-g(x)$ не возрастает по $x$, и, следовательно, отношение $\bar{G}(x+u) / \bar{G}(x)=e^{-(g(x+u)-g(x))}$ не убывает по $x$. Поэтому функция

$$
J_{t}(x)=\frac{\overline{G_{t}}(x)}{\bar{G}(x)}=\int_{0}^{t} \frac{\bar{G}(x+u)}{\bar{G}(x)} d u
$$

также не убывает по $x$. Отсюда, поскольку $\overline{G_{t}}(x)=J_{t}(x) \bar{G}(x)$, значение второго интеграла в (9) допускает оценку

$$
\begin{aligned}
& \int_{U}^{x} \frac{J_{t}(x-u)}{J_{t}(x)} \frac{\bar{G}(x-u)}{\bar{G}(x)} d G_{t}(u) \leqslant \int_{U}^{x} \frac{\bar{G}(x-u)}{\bar{G}(x)} d G_{t}(u) \\
& \quad=\int_{U}^{x} \frac{\bar{G}(x-u)}{\bar{G}(x)}(\bar{G}(u)-\bar{G}(u+t)) d u \leqslant \int_{U}^{x} \frac{\bar{G}(x-u) \bar{G}(u)}{\bar{G}(x)} d u
\end{aligned}
$$

и ввиду условия (14) может быть сделано сколь угодно малым выбором достаточно большого $U$. Отсюда по лемме 2 вытекает сильная субэкспоненциальность распределения $G$.

Распределение Парето с параметром $\alpha>1$, равно как и любое распределение с правильно меняющимся на бесконечности хвостом и с конечным средним значением, удовлетворяет условиям леммы 4 и, следовательно, является сильно субэкспоненциальным.

Распределение Вейбулла $\bar{G}(x)=e^{-x^{\alpha}}, \alpha \in(0,1)$, удовлетворяет условиям леммы 5. Действительно, функция $g(x)=x^{\alpha}$ вогнута при $\alpha \in(0,1)$, и осталось проверить выполнение условия (14). Функция $(x-u)^{\alpha}+u^{\alpha}$ достигает своего минимума по $u \in[U, x-U]$ на концах этого отрезка, следовательно,

$$
\int_{U}^{x-U} \bar{G}(x-u) \bar{G}(u) d u=\int_{U}^{x-U} e^{-\left((x-u)^{\alpha}+u^{\alpha}\right)} d u \leqslant x e^{-\left((x-U)^{\alpha}+U^{\alpha}\right)}=o\left(e^{-x^{\alpha}}\right)
$$

например, при $U=U(x)=\ln ^{2 / \alpha} x$. Кроме того, при таком выборе $U(x)$

$$
\left(\int_{0}^{U}+\int_{x-U}^{x}\right) \bar{G}(x-u) \bar{G}(u) d u \sim \bar{G}(x) \int_{0}^{\infty} \bar{G}(u) d u .
$$

Из последних двух соотношений вытекает (14).

Примерно так же проверяется, что и логнормальное распределение удовлетворяет условиям леммы 5 и, следовательно, является сильно субэкспоненциальным.

4. Некоторые свойства сверток сильно субэкспоненциального распределения. Пусть $G$ - сильно субэкспоненциальное распределение в $\mathbf{R}^{+}$. Настоящий пункт содержит аналоги стандартных свойств субэкспоненциальных распределений для распределений класса $\mathscr{S}_{*}$.

Лемма 6. Для мюбого натурального $k$ асимптотика хвоста $k$-й свертки распределения $G_{t}$ имеет вид $\overline{G_{t}^{k *}}(x) \sim k \overline{G_{t}}(x)$ при $x \rightarrow \infty$ равномерно по $t \geqslant 1$.

Д о к а з а те л ь с в о следует по индукции из равенства для хвоста свертки $G_{t}^{(k+1) *}$

и из леммы 2.

$$
\overline{G_{t}^{(k+1) *}}(x)=\left(\int_{0}^{U}+\int_{U}^{x}\right) \overline{G_{t}^{k *}}(x-u) d G_{t}(u)+\overline{G_{t}}(x)
$$

Докажем следующую оценку для хвоста $k$-й свертки меры $G_{t}$. 
Лемма 7. Для любого $\varepsilon>0$ суиествует $c=c(\varepsilon)$ maкое, ито при яюбых $x \geqslant 0$, $t \geqslant 1 u k=1,2, \ldots$ имеет место неравенство

$$
\overline{G_{t}^{k *}}(x) \leqslant c \overline{G_{t}}(x)(1+\varepsilon)^{k} .
$$

Д ока за те ль с т в о. Пусть $\varepsilon>0$. Ввиду сильной субэкспоненциальности распределения $G$ из (8) вытекает существование числа $x_{0}=x_{0}(\varepsilon)$ такого, что при любых $x \geqslant x_{0}$ и $t \geqslant 1$

$$
\int_{0}^{x} \frac{\overline{G_{t}}(x-u)}{\overline{G_{t}}(x)} d G_{t}(u) \leqslant 1+\varepsilon .
$$

Обозначим $A_{k} \equiv \sup _{x \geqslant 0, t \geqslant 1}\left[\overline{G_{t}^{k *}}(x) / \overline{G_{t}}(x)\right]$. Оценим сверху $A_{k+1}$ через $A_{k}$. В силу (15)

$$
A_{k+1} \leqslant \sup _{x \geqslant 0, t \geqslant 1} \int_{0}^{x} \frac{\overline{G_{t}^{k *}}(x-u)}{\overline{G_{t}}(x)} d G_{t}(u)+1 .
$$

По определению $A_{k}$ имеем неравенство

$$
\int_{0}^{x} \frac{\overline{G_{t}^{k *}}(x-u)}{\overline{G_{t}}(x)} d G_{t}(u)=\int_{0}^{x} \frac{\overline{G_{t}^{k *}}(x-u)}{\overline{G_{t}}(x-u)} \frac{\overline{G_{t}}(x-u)}{\overline{G_{t}}(x)} d G_{t}(u) \leqslant A_{k} \int_{0}^{x} \frac{\overline{G_{t}}(x-u)}{\overline{G_{t}}(x)} d G_{t}(u) .
$$

Ввиду (16) имеем отсюда верную при $x \geqslant x_{0}$ оценку

$$
\int_{0}^{x} \frac{\overline{G_{t}^{k *}}(x-u)}{\overline{G_{t}}(x)} d G_{t}(u) \leqslant A_{k}(1+\varepsilon) .
$$

Кроме того, при $x<x_{0}$ и $t \geqslant 1$

$$
\int_{0}^{x} \frac{\overline{G_{t}^{k *}}(x-u)}{\overline{G_{t}}(x)} d G_{t}(u) \leqslant \frac{1}{\overline{G_{t}}\left(x_{0}\right)} \leqslant \frac{1}{\min \left(1, \bar{G}\left(x_{0}+1\right)\right)} \equiv c_{1}\left(x_{0}\right)<\infty .
$$

Из двух последних оценок следует, что для любых $x \geqslant 0$ и $t \geqslant 1$

$$
\int_{0}^{x} \frac{\overline{G_{t}^{k *}}(x-u)}{\overline{G_{t}}(x)} d G_{t}(u) \leqslant A_{k}(1+\varepsilon)+c_{1} .
$$

Подставляя эту оценку в (17), получаем $A_{k+1} \leqslant A_{k}(1+\varepsilon)+c_{1}+1$. Отсюда вытекает неравенство $A_{k+1} \leqslant\left(c_{1}+1\right)(k+1)(1+\varepsilon)^{k}$, которое эквивалентно оценке леммы.

5. Оценка сверху для хвоста распределения первой до момента времени $n$ неотрицательной суммы. Пусть $\eta=\min \left\{k \geqslant 1: S_{k} \geqslant 0\right\}-$ номер первой неотрицательной лестничной высоты (считаем $\min \varnothing=\infty), \eta^{[n]}=\min \{k \in[1, n]$ : $\left.S_{k} \geqslant 0\right\}$ - номер первой до момента времени $n$ неотрицательной лестничной высоты и $\chi^{[n]}=S_{\eta[n]}$ - первая до момента времени $n$ неотрицательная сумма.

Поскольку $\mathbf{E} \xi<0$, то $\eta, \eta^{[n]}$ и $\chi^{[n]}-$ несобственные случайные величины; положим $\boldsymbol{p}=\mathbf{P}\{\eta<\infty\}, p^{[n]}=\mathbf{P}\left\{\eta^{[n]}<\infty\right\}$. При любом $n$ справедливы неравенства

$$
0<\mathbf{P}\{\xi \geqslant 0\} \leqslant p^{[n]} \leqslant p<1 .
$$

Кроме того,

$$
p^{[n]} \uparrow p \quad \text { при } n \rightarrow \infty .
$$

Справедлива следующая оценка сверху для вероятности события $\left\{\chi^{[n]} \geqslant x\right\}$ при больших значениях $n$ и $x$.

Лемма 8. Пусть распределекие $F$ локально степекное. Тогда дяя любого $\varepsilon>0$ найдутся числа $n_{1}$ u $x_{1}$ тахие, что при $n \geqslant n_{1}$ u $x \geqslant x_{1}$ справедливо керавенство

$$
\mathbf{P}\left\{\chi^{[n]} \geqslant x\right\} \leqslant(1+\varepsilon) \frac{1-p}{a} \int_{x}^{x+n a} \bar{F}(u) d u .
$$


Д о к а $з$ а т е л ь с т в о. По формуле полной вероятности имеем

$$
\mathbf{P}\left\{\chi^{[n]} \geqslant x\right\}=\sum_{j=1}^{n} \mathbf{P}\left\{S_{i}<0 \text { при всех } i \leqslant j-1, S_{j} \geqslant x\right\} .
$$

Положим $\psi_{j}(B)=\mathbf{P}\left\{S_{i}<0\right.$ при всех $\left.i \leqslant j, S_{j} \in B\right\}, B \subseteq(-\infty, 0)$. По формуле полной вероятности справедливо равенство

$$
\mathbf{P}\left\{S_{i}<0 \text { при всех } i \leqslant j-1, S_{j} \geqslant x\right\}=\int_{x}^{\infty} F(d y) \psi_{j-1}([x-y, 0)) .
$$

Подставляя это равенство в (20), получаем

$$
\mathbf{P}\left\{\chi^{[n]} \geqslant x\right\}=\int_{x}^{\infty} F(d y) \sum_{j=1}^{n} \psi_{j-1}([x-y, 0)) .
$$

Оценим сумму в последнем представлении. Для любого $N<n$

$$
\begin{aligned}
& \sum_{j=1}^{n} \psi_{j-1}([x-y, 0)) \leqslant N+\sum_{j=N+1}^{n} \mathbf{P}\left\{S_{1}<0, \ldots, S_{N}<0, S_{j} \geqslant x-y\right\} \\
& \quad=N+\mathbf{P}\left\{S_{1}<0, \ldots, S_{N}<0\right\} \sum_{j=N+1}^{n} \mathbf{P}\left\{S_{j} \geqslant x-y \mid S_{1}<0, \ldots, S_{N}<0\right\} .
\end{aligned}
$$

Поскольку

$$
\mathbf{P}\left\{S_{1}<0, \ldots, S_{N}<0\right\} \longrightarrow 1-p \text { при } N \rightarrow \infty,
$$

то для любого $\delta>0$ найдется $N$ такое, что

$$
\begin{aligned}
\sum_{j=1}^{n} \psi_{j-1}([x-y, 0)) & \leqslant N+(1-p+\delta) \sum_{j=N+1}^{n} \mathbf{P}\left\{S_{j} \geqslant x-y \mid S_{1}<0, \ldots, S_{N}<0\right\} \\
& \leqslant N+(1-p+\delta) \sum_{j=N+1}^{\infty} \mathbf{P}\left\{S_{j} \geqslant x-y \mid S_{1}<0, \ldots, S_{N}<0\right\} .
\end{aligned}
$$

Асимптотика функции восстановления не зависит от значений первых $N$ слагаемых. Поэтому для любого фиксированного $N$ в силу теоремы восстановления для любого $\delta>0$ найдется $t$ такое, что при $y-x \geqslant t$

$$
\sum_{j=N+1}^{\infty} \mathbf{P}\left\{S_{j-1} \geqslant x-y \mid S_{1}<0, \ldots, S_{N}<0\right\} \leqslant(1+\delta) \frac{y-x}{a} .
$$

Кроме того, при $y \in[x, x+t)$ справедлива оценка

$$
\begin{aligned}
& \sum_{j=N+1}^{\infty} \mathbf{P}\left\{S_{j-1} \geqslant x-y \mid S_{1}<0, \ldots, S_{N}<0\right\} \\
& \quad \leqslant \sum_{j=N+1}^{\infty} \mathbf{P}\left\{S_{j-1} \geqslant-t \mid S_{1}<0, \ldots, S_{N}<0\right\}=\tilde{c}=\tilde{c}(-t)<\infty .
\end{aligned}
$$

Из двух последних оценок вытекает неравенство, верное при любом $y \geqslant x(\widehat{c}=N+\tilde{c})$ :

$$
\sum_{j=1}^{n} \psi_{j-1}([x-y, 0)) \leqslant(1-p+\delta)(1+\delta) \frac{y-x}{a}+\widehat{c} .
$$

При любом $y \geqslant x$ имеем также следующие неравенство и асимптотику

$$
\sum_{j=1}^{n} \psi_{j-1}([x-y, 0)) \leqslant \sum_{j=1}^{n} \mathbf{P}\left\{S_{1}<0, S_{2}<0, \ldots, S_{j}<0\right\} \sim n(1-p)
$$


при $n \rightarrow \infty$ ввиду (22). Следовательно, при достаточно больших $n$

$$
\sum_{j=1}^{n} \psi_{j-1}([x-y, 0)) \leqslant n(1-p+\delta) .
$$

Обозначим $g(x, y) \equiv(1-p+\delta) \min ((1+\delta)(y-x) / a, n)$. Подставляя оценки (23) и (24) в (21), приходим к неравенству

$$
\begin{aligned}
\mathbf{P}\left\{\chi^{[n]} \geqslant x\right\} \leqslant \widehat{c} \int_{x}^{\infty} F(d y)+\int_{x}^{\infty} F(d y) g(x, y)=\widehat{c} \bar{F}(x)-\left.\bar{F}(y) g(x, y)\right|_{x} ^{\infty} \\
\quad+\int_{x}^{\infty} \bar{F}(y) d_{y} g(x, y)=\widehat{c} \bar{F}(x)+(1-p+\delta) \frac{1+\delta}{a} \int_{x}^{x+n a /(1+\delta)} \bar{F}(y) d y .
\end{aligned}
$$

Поскольку функция $\bar{F}(x)$ локально степенная, то $\bar{F}(x)=o\left(\int_{x}^{x+n a /(1+\varepsilon)} \bar{F}(y) d y\right)$ при $n, x \rightarrow \infty$. Поэтому из (25) вытекает утверждение леммы.

6. Оценка сверху для вероятностей больших уклонений максимума сумм. Определим собственную случайную величину $\tilde{\chi}^{[n]}$ с распределением

$$
\mathbf{P}\left\{\tilde{\chi}^{[n]} \in B\right\}=\mathbf{P}\left\{\chi^{[n]} \in B\right\}\left(p^{[n]}\right)^{-1}, \quad B \subseteq[0, \infty) .
$$

Пусть $\varepsilon>0$; положим $b=(1+\varepsilon)(1-p) / p a$. В силу леммы 8 и сходимости (19) существуют $n_{0}$ и $x_{0}$ такие, что для любых $n \geqslant n_{0}$ и $x \geqslant x_{0}$

$$
\mathbf{P}\left\{\tilde{\chi}^{[n]} \geqslant x\right\} \leqslant b \int_{x}^{x+n a} \bar{F}(u) d u .
$$

Определим вероятностную меру $G$ на полупрямой $\mathbf{R}^{+}$, положив

$$
\bar{G}(x)=\min (1, b \bar{F}(x)) \text { при } x \geqslant x_{0}+1, \quad \bar{G}\left(x_{0}+1\right)=1 .
$$

Тогда в силу (26) при $n \geqslant n_{0}$ и $x \geqslant 0$

$$
\mathbf{P}\left\{\tilde{\chi}^{[n]} \geqslant x\right\} \leqslant \overline{G_{n a}}(x) .
$$

Пусть $\tilde{\chi}_{1}^{[n]}, \tilde{\chi}_{2}^{[n]}, \ldots$ - суть независимые копии случайной величины $\tilde{\chi}^{[n]}$. Одна из сумм $S_{i}, 1 \leqslant i \leqslant n$, превосходит уровень $x$ лишь тогда, когда одна из лестничных высот превзошла этот уровень. Вероятность того, что $i$-я лестничная высота существует, причем до момента времени $n$, не превосходит $\left(p^{[n]}\right)^{i}$. Обозначим через $\widehat{p}_{i}[n]$ условную вероятность того, что $i$-я лестничная высота окажется последней при условии, что она существует до момента времени $n$. Для любого фиксированного $i$ имеем монотонную сходимость

$$
\widehat{p}_{i}^{[n]} \downarrow 1-p \quad \text { при } n \rightarrow \infty .
$$

По формуле полной вероятности для любых $x$ и $n$ имеем неравенство:

$$
\mathbf{P}\left\{\max _{0 \leqslant i \leqslant n} S_{i} \geqslant x\right\} \leqslant \sum_{i=1}^{n}\left(p^{[n]}\right)^{i} \widehat{p}_{i}^{[n]} \mathbf{P}\left\{\tilde{\chi}_{1}^{[n]}+\cdots+\tilde{\chi}_{i}^{[n]} \geqslant x\right\} .
$$

Пусть $N<n$. Разбивая последнюю сумму на две и используя (18) и неравенства $\widehat{p}_{i}^{[n]} \leqslant \widehat{p}_{i+1}^{[n]}$ и $(28)$, получаем оценку

$$
\mathbf{P}\left\{M_{n} \geqslant x\right\} \leqslant \widehat{p}_{N}^{[n]} \sum_{i=1}^{N} p^{i} \overline{G_{n a}^{i *}}(x)+\sum_{i=N+1}^{\infty} p^{i} \overline{G_{n a}^{i *}}(x),
$$

верную для локально степенного распределения $F$.

Далее считаем, что случайная величина $\boldsymbol{\xi}$ имеет сильно субэкспоненциальное распределение. Соответственно, в силу определения (27) и леммы 3 распределение $G$ также сильно субэкспоненциальное. Имеет место

Лемма 9. Пусть $F \in \mathscr{S}_{*}$. Тогда для любого $\varepsilon>0$ суиествует $x_{1}$ mакое, ито при всех $n \geqslant 1$ и $x \geqslant x_{1}$ справедлива очекка

$$
\mathbf{P}\left\{M_{n} \geqslant x\right\} \leqslant \frac{1+\varepsilon}{a} \int_{x}^{x+n a} \bar{F}(u) d u .
$$


Д о к а з а т е л ь с т в о. Поскольку временной параметр $n$ принимает лишь счетное число значений и функция $\bar{F}(u)$ локально степенная, то достаточно проверить следующие два соотношения: при любом фиксированном $n$

$$
\lim _{x \rightarrow \infty} \frac{\mathbf{P}\left\{M_{n} \geqslant x\right\}}{\bar{F}(x)}=\frac{n}{a}, \quad \limsup _{n, x \rightarrow \infty} \frac{\mathbf{P}\left\{M_{n} \geqslant x\right\}}{\int_{x}^{x+n a} \bar{F}(u) d u} \leqslant \frac{1}{a} .
$$

Tак как распределение $F$ субэкспоненциальное, то равенство (3) позволяет проверить первое соотношение в (31) по индукции. Действительно, при $n=1$ имеем $X_{1}=\xi_{1}^{+}$и $\mathbf{P}\left\{X_{1} \geqslant x\right\}=\bar{F}(x)$ при $x>0$. Поскольку $\mathbf{P}\left\{X_{n+1} \geqslant x\right\}=\mathbf{P}\left\{X_{n}+\xi_{n+1} \geqslant x\right\}$ при $x>0$, индукционный переход следует из стандартных свойств субэкспоненциальных распределений (см., например, доказательство теоремы 1 в [4] и предложения 1 в [6]).

Перейдем к проверке второго соотношения в (31), исходя из оценки (30). Распределение $G$ сильно субэкспоненциальное, поэтому по лемме 7 для любого $\delta>0$ существует $c_{1}$ такое, что

$$
\limsup _{x \rightarrow \infty} \frac{1}{\overline{G_{n a}}(x)} \sum_{i=N+1}^{\infty} p^{i} \overline{G_{n a}^{i *}}(x) \leqslant \frac{c_{1}[p(1+\delta)]^{N+1}}{1-p(1+\delta)}
$$

равномерно по $n \geqslant 1$. Подставляя это неравенство в (30), а также используя (29) и лемму 6, получаем

$$
\limsup _{n, x \rightarrow \infty} \frac{\mathbf{P}\left\{M_{n} \geqslant x\right\}}{\overline{G_{n a}}(x)} \leqslant(1-p) \sum_{i=1}^{N} p^{i} i+\frac{c_{1}[p(1+\delta)]^{N+1}}{1-p(1+\delta)} .
$$

Отсюда ввиду произвольности выбора $\delta$ и $N$ вытекает неравенство

$$
\limsup _{n, x \rightarrow \infty} \frac{\mathbf{P}\left\{M_{n} \geqslant x\right\}}{\overline{G_{n a}}(x)} \leqslant \frac{p}{1-p},
$$

следствием которого в силу определения $G$ является соотношение

$$
\limsup _{n, x \rightarrow \infty} \frac{\mathbf{P}\left\{M_{n} \geqslant x\right\}}{\int_{x}^{x+n a} \bar{F}(u) d u} \leqslant \frac{1+\varepsilon}{a} .
$$

Тах как $\varepsilon>0$ выбрано произвольно, то справедливо второе соотношение в (31). Лемма доказана.

\section{СПИСОК ЛИТЕРАТУРЫ}

1. Годоваячух В.В. Вероятности больших уклонений для сумм независимых случайных величин, принадлежащих области притяжения устойчивого закона. Теория вероятн. и ее примен., 1978, т. 23, в. 3, с. 624-630.

2. Пияелис И. Ф. Одна задача о больших уклонениях в пространстве траекторий. Теория вероятн. и ее примен., 1981, т. 26, в. 1, с. 73-87.

3. Феллер В. Введение в теорию вероятностей и ее приложения. Т. 2. М.: Мир, 1984, $752 \mathrm{c}$.

4. Чистяжов В.П. Теорема о суммах независимых положительных случайных величин и ее приложения к ветвящимся случайным процессам. - Теория вероятн. и ее примен., 1964 , т. 9 , в. 4 , с. $710-718$.

5. Боровков A.A., Боровков K.A. О вероятностях больших уклонений для случайных блужданий. I. Распределения с правильно меняющимися хвостами. Теория вероятн. и ее примен., 2001, т. 46, в. 2, с. 209-232.

6. Embrechts P., Goldie C.M., Veraverbeke N. Subexponentiality and infinite divisibility. - Z. Wahrscheinlichkeitstheor. verw. Geb., 1979, B. 49, S. 335-347.

7. Klüppelberg C. Subexponential distributions and integrated tails. - J. Appl. Probab., 1988 , v. 25 , № 1, p. 132-141. 
8. Korshunov D. A. On distribution tail of the maximum of a random walk. - Stochastic Process. Appl., 1997, v. 72, № 1, p. 97-103.

9. Sgibnev M.S. On the distribution of the maxima of partial sums. - Statist. Probab. Lett., 1996, v. 28, № 3, p. 235-238.

10. Teugels J. L. The class of subexponential distributions. - Ann. Probab., 1975, v. 3, № 6, p. 1000-1011.

11. Veraverbeke $N$. Asymptotic behavior of Wiener-Hopf factors of a random walk. Stochastic Process. Appl., 1977, v. 5, № 1, p. 27-37.

Поступила в редакцию 19.X.1998

(C) $2001 \mathrm{r}$.

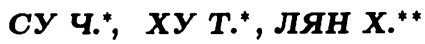

\section{О ЛОГАРИФМИЧЕСКОМ ЗАКОНЕ ДЛЯ СТРОГО СТАЦИОНАРНЫХ ОТРИЦАТЕЛЬНО АССОЦИИРОВАННЫХ СЕРИЙ ${ }^{1)}$}

Доказан логарифмический закон для строго стационарных и отрицательно ассоциированных серий случайных величин, аналогичный логарифмическому закону для независимых одинаково распределенных случайных величин. Использована формула Парсеваля из теории преобразований Фурье для оценки близости между отрицательно ассоциированными и независимыми случайными величинами. Подчеркивается польза применения использованных методов для дохазательства предельных теорем для отрицательно ассоциированных случайных величин.

Ключевые слова и фразы: отрицательно ассоциированная последовательность, серия; логарифмический закон, формула Парсеваля, преобразование Фурье.

0. Введение. Семейство случайных величин $\left\{X_{i}, i=1, \ldots, n\right\}$ называется отричательно ассочиированяым (NA), если для любых двух непересекающихся непустых подмножеств $A$ и $B$ множества $\{1,2, \ldots, n\}$ выполняется неравенство

$$
\operatorname{cov}\left(f_{1}\left(X_{i}, i \in A\right), f_{2}\left(X_{j}, j \in B\right)\right) \leqslant 0,
$$

где $f_{1}$ и $f_{2}$ - покоординатно возрастающие функции такие, что ковариация существует.

Бесконечное множество случайных величин называется отрицательно ассоциированным, если любое конечное его подмножество обладает этим свойством. Свойство множества быть отрицательно ассоциированным также называют отрицательяой ассочиนроваяностью. Это определение было введено Аламом и Сахсеном [1], а затем детально изучалось Йо-Девом и Прошаном [3]. Понятие NA привлекает все большее

Su Chun, Hu Taizhong, Liang Hanying. On the logarithm law for strictly stationary and negatively associated arrays.

(C) Перевод на русский язык, Научное издательство «ТВП», 2001.

* Department of Statistics and Finance, University of Science and Technology of China, Hefei, Anhui 230026, China; e-mail: suchun@ustc.edu.cn, thu@ustc.edu.cn

** Department of Applied Mathematics Tongji University, Shanghai 200092, China.

1) Работа выполнена при поддержке NSF (гранты 19671078 и 19701030), the Doctoral Program Foundation of Institute of High Education и Chinese Academy of Sciences. 